\title{
On the edge-density of 4-critical graphs
}

Mathematics Subject Classification (2000): 05C15

\author{
Babak Farzad ${ }^{1}$ \\ Department of Mathematics, Brock University \\ Michael Molloy ${ }^{2}$ \\ Department of Computer Science, University of Toronto
}

\author{
Manuscript correspondence: \\ e-mail: bfarzad@brocku.ca \\ telephone: (905) 6885550 ext. 3145 \\ fax: (905) 3785713 Attn. Babak Farzad \\ mailing address: Babak Farzad \\ Department of Mathematics \\ Brock University \\ 500 Glenridge Ave. \\ St. Catharines, Ontario, L2S 3A1 \\ CANADA
}

\footnotetext{
${ }^{1}$ Much of this research was done when the author was a graduate student at the University of Toronto and a postdoctoral fellow at McGill University. This research is supported by NSERC and University of Toronto Fellowship.

${ }^{2}$ This research is supported in part by NSERC and a Sloan Research Fellowship.
} 


\title{
On the edge-density of 4-critical graphs
}

\author{
Babak Farzad Michael Molloy
}

\begin{abstract}
Gallai conjectured that every 4-critical graph on $n$ vertices has at least $\frac{5}{3} n-\frac{2}{3}$ edges. We prove this conjecture for 4 -critical graphs in which the subgraph induced by vertices of degree 3 is connected.
\end{abstract}

\section{Introduction}

For a graph $G=(V, E)$, let $|G|,\|G\|, \chi(G)$ denote the number of vertices, the number of edges and the chromatic number of $G$, respectively. Also, let $\operatorname{deg}_{G}(v)$ denote the degree of vertex $v$ in $G$. The set of neighbours of a vertex $v$ in $G$ is denoted by $N_{G}(v)$. More generally for $U \subseteq V$, the set of neighbours in $V \backslash U$ of vertices in $U$ is denoted by $N(U)$. A graph $G$ is called critical if $\chi(H)<\chi(G)$ for every proper subgraph $H$ of $G$; it is called $k$-critical if it is critical and $\chi(G)=k$. In 1963, Gallai [Gal63] conjectured the following:

Conjecture 1.1 For each $k \geq 4$, for every $k$-critical graph $G$,

$$
\|G\| \geq \frac{k-1}{2}|G|+\frac{(k-3)(|G|-k)}{2(k-1)} .
$$

For $k=4$, this conjecture states that every 4-critical graph $G$ has at least $\frac{5}{3}|G|-\frac{2}{3}$ edges.

The low-vertex subgraph of a $k$-critical graph is the subgraph induced by the vertices of degree $k-1$, i.e. the vertices of minimum degree. The 
low-vertex subgraph was defined by Gallai in his first study of critical graphs [Gal63] where he proved the important structural result that every block of the low-vertex subgraph is either a complete graph or an odd cycle. It has since become a crucial part of the study of critical graphs. For example, most known lower bounds on the number of edges in $k$-critical graphs are based on Gallai's characterization of the low-vertex subgraph.

In this paper, we prove Gallai's conjecture for every 4-critical graph whose low-vertex subgraph is connected:

Theorem 1.2 For every 4-critical graph $G$ with a connected low-vertex subgraph, $\|G\| \geq \frac{5}{3}|G|-\frac{2}{3}$.

\section{$1.1 \quad$ Related work}

The lower bound from Conjecture 1.1 is the best possible. One can see this by applying Hajos' Construction (see [Kri98]) to make an infinite family $\mathcal{F}$ of $k$-critical graphs, $k \geq 3$, for which the bound is tight. We start by $\mathcal{F}=\left\{K_{k}\right\}$ for which it is easy to see that the the bound is tight. Repeatedly, we extend $\mathcal{F}$ in the following way. Let $G_{1}=\left(V_{1}, E_{1}\right)$ and $G_{2}=\left(V_{2}, E_{2}\right)$ be two disjoint copies of $k$-critical graphs in $\mathcal{F}$, and let $e_{1}=\left(u_{1}, v_{1}\right) \in E_{1}$ and $e_{2}=\left(u_{2}, v_{2}\right) \in E_{2}$. Denote by $G$ the graph obtained from $G_{1}$ and $G_{2}$ by applying Hajos' Construction as follows: (i) delete $e_{1}$ and $e_{2}$; (ii) identify $u_{1}$ and $u_{2}$; (iii) join $v_{1}$ and $v_{2}$ by an edge. It is easy to see that $G$ is $k$-critical and that the lower bound from Conjecture 1.1 is tight for $G$. Thus we add $G$ to $\mathcal{F}$.

In the remainder of this section, we will assume that $n$ is the number of vertices of the critical graph and that $k \geq 4$, as 1-, 2- and 3-critical graphs 
have a very simple structure.

The fact that every vertex of a $k$-critical graph has degree at least $k-1$, easily implies that every $k$-critical graph has at least $\frac{(k-1) n}{2}$ edges. Brooks' Theorem strengthens this slightly to $\frac{(k-1) n}{2}+\frac{1}{2}$ when the graph is not $K_{k}$, since at least one vertex has degree at least $k$. Dirac [Dir57] proved that every $k$-critical graph, other than $K_{k}$, the complete graph on $k$ vertices, has at least $\frac{(k-1) n}{2}+\frac{k-3}{2}$ edges. This bound is tight, as the following example shows: consider a graph consisting of a $(k-2)$-clique, $A$, a $(k-1)$-clique, $B$ and two non-adjacent vertices $u, v$ which are both adjacent to every vertex in $A$, and whose neighbourhoods in $B$ define a non-trivial partition of $B$.

Weinstein [Wei75] gave a shorter proof for Dirac's lower bound by showing that any counterexample is $(k-1)$-regular and thus contradicts Brooks' Theorem. ${ }^{3}$ Later, Mitchem [Mit78] gave another proof for this bound where he also showed that the only graphs for which it is tight have the same structure as the example given above. Dirac [Dir74] extended his bound by showing that for $k \geq 5$, if a $k$-critical graph is not $K_{k}$ or one of those with the same structure as the example above, then it has at least $\frac{(k-1) n}{2}+\frac{k-1}{2}$ edges. Kostochka and Stiebitz [KS99] strengthened this to $\frac{(k-1) n}{2}+(k-3)$ for any $k \geq 4$, so long as the graph is not $K_{k}$ and $n \neq 2 k-1$.

As an easy implication of his characterization of the low-vertex subgraph, Gallai (see [JT95]) proved that if a $k$-critical graph is not $K_{k}$, then it has at least $\frac{(k-1) n}{2}+\frac{(k-3) n}{2\left(k^{2}-3\right)}$ edges. In particular, the number of edges in a 4 critical graph (other than $K_{4}$ ) is at least $\frac{20}{13} n$. Kostochka and Stiebitz [KS98]

\footnotetext{
${ }^{3} \mathrm{~A}$ completely different proof was given later by Kronk and Mitchem [KM72] which was motivated by Melnikov and Vizing's proof of Brooks' theorem.
} 
improved that bound, in the case where $n \neq 2 k-1$, to $\frac{(k-1) n}{2}+\frac{(k-3) n}{2\left(k^{2}-3\right)}+\frac{k-4}{2}$. Krivelevich [Kri98] improved it further to $\frac{(k-1) n}{2}+\frac{(k-3) n}{2\left(k^{2}-2 k-1\right)}$ (even when $n=2 k-1$ ). For $k=4$ then Krivelevich's bound is $\frac{11}{7} n$ which is currently the best known.

Recently, Kostochka and Stiebitz [KS03] proved that for $k \geq 6$, every $k$-critical graph other than $K_{k}$ has at least $\frac{(k-1) n}{2}+\frac{(k-3) n}{2((k-c)(k-1)+k-3)}$ edges where $c=(k-5)\left(\frac{1}{2}-\frac{1}{(k-1)(k-2)}\right)$. This improves Krivelevich's bound for $k \geq 6$.

The high-vertex subgraph of a $k$-critical graph is the subgraph induced by the vertices of degree at least $k$; i.e. it is what remains after deleting the low-vertex subgraph. A key ingredient in Krivelevich's proof is the following theorem of Stiebitz [Sti82], which was first conjectured by Gallai.

Theorem 1.3 In every $k$-critical graph with at least one vertex of degree $k-1$, the low-vertex subgraph has at least as many components as the highvertex subgraph.

This immediately implies the following lower bound on $e_{H}$, the number of edges in $H$, the high-vertex subgraph. We use $r$ to denote the number of components of the low-vertex subgraph:

$$
\|H\| \geq|H|-r
$$

Any improvement on (1) will yield an improvement on Krivelevich's bound. Most of the work in this paper can be viewed as obtaining substantial improvement for the case $r=1$, i.e. when the low-vertex subgraph is connected. 


\subsection{Definitions and preliminaries}

It is easy to see that every vertex of a $k$-critical graph has degree at least $k-1$. The vertices of a $k$-critical graph $G$ whose degrees are $k-1$ are called low vertices, and the others are called high vertices. The subgraphs of $G$ induced by the set of low vertices and the set of high vertices are called the low-vertex subgraph $L(G)$ and the high-vertex subgraph $H(G)$, respectively.

Define a block to be a maximal 2-connected subgraph of a graph $G$. A connected graph all of whose blocks are either complete graphs or odd cycles is called a Gallai tree. A graph all of whose components are Gallai trees is called a Gallai forest. A $k$-Gallai tree (forest) is a Gallai tree (forest) in which all vertices have degree at most $k-1$. Gallai [Gal63] proved the following important structural result on critical graphs.

Theorem 1.4 If $G$ is a $k$-critical graph then the low-vertex subgraph of $G$ is a $k$-Gallai forest.

Consider any $k$-colouring $\phi$ of $A \subset G$ and any $B \subseteq G-A$. A $k$-colouring of $A \cup B$ is an extension of $\phi$ if it agrees on $A$. A vertex $v$ of a 4 -Gallai tree $L$ is called a $d$-vertex if it is contained in no cycle in $L$.

Gallai's proof of Theorem 1.4 implies a more general statement:

Lemma 1.5 Suppose that $L$ is a connected subgraph of an arbitrary graph $G$ induced by a subset of the vertices of degree $k-1$ (in $G$ ). If there is a $(k-1)$-colouring of $G-L$ that has no extension to $L$, then $L$ is a $k$-Gallai tree. Moreover, if $k=4$ and $B$ is any component of what remains of $L$ after deleting all of the d-vertices then all vertices of $N(B)$ in $G-L$ have the same colour. 
We use $L$ and $H$ instead of $L(G)$ and $H(G)$, respectively, when there is no ambiguity. Let $n_{d}$ denote the number of $d$-vertices. Also, let $\|L, H\|$ denote the number of edges with exactly one endpoint in $L$.

For the remainder of this section, we will assume that $G$ is a 4 -critical graph with a connected low-vertex subgraph.

Lemma 1.6 If $|H| \geq 1$ then $\|L\|+\|L, H\| \geq \frac{5}{3}|L|+1+\frac{n_{d}}{3}$.

Proof. Let $c$ denote the number of cycles in $L$. Since $L$ is a 4 -Gallai tree other than $K_{4}$, we know that $\|L\|=|L|-1+c$. Moreover, $\|L, H\|=$ $\sum_{v \in V(L)} \operatorname{deg}_{G}(v)-\sum_{v \in V(L)} \operatorname{deg}_{L}(v)=|L|+2-2 c$. This implies that $\|L\|+$ $\|L, H\|=2|L|+1-c \geq 2|L|+1-\frac{|L|-n_{d}}{3}=\frac{5}{3}|L|+1+\frac{n_{d}}{3}$.

Let a >3-cycle denote a cycle of length at least four. The above proof shows that the bound in the lemma is tight iff there is no $>3$-cycle in the lowvertex subgraph. In fact, Construction 1.8 below shows that for 4-critical graphs, this bound is tight in the sense that from any 4-critical graph $G$ with $>3$-cycles in its low-vertex subgraph, we can get another 4-critical graph $G^{\prime}$ with $H(G)=H\left(G^{\prime}\right)$ where $L\left(G^{\prime}\right)$ has no $>3$-cycle, and $\|G\| \geq \frac{5}{3}|G|-\frac{2}{3}$ if $\left\|G^{\prime}\right\| \geq \frac{5}{3}\left|G^{\prime}\right|-\frac{2}{3}$. This indicates that obtaining the appropriate lower bound on the number of edges of the high-vertex subgraph, $\|H(G)\|$, is the main bottleneck in getting to Gallai's Conjecture. Since by the classical theorem of Brooks, $K_{4}$ is the only 4-critical graph with empty high-vertex subgraph, an immediate implication of Lemma 1.6 is

Corollary 1.7 If $|H| \leq 1$ then $\|G\| \geq \frac{5}{3}|G|-\frac{2}{3}$.

In order to prove Theorem 1.2, considering Lemma 1.6 and Corollary 1.7, 

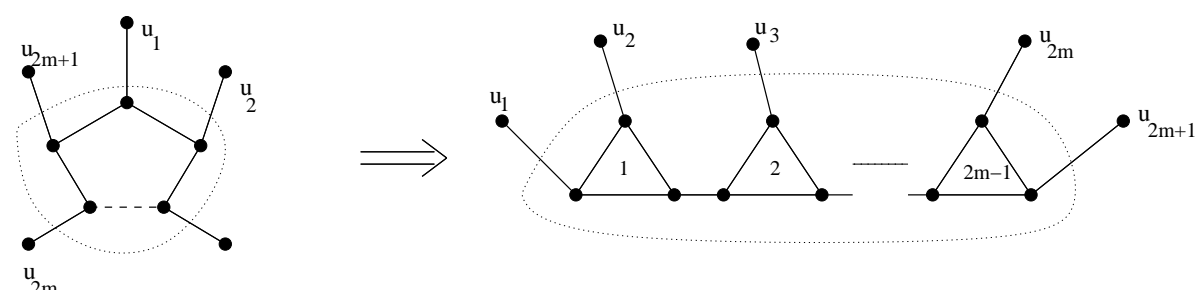

Figure 1: Replacing a $(2 m+1)$-cycle $C$ in $L(G)$ with a sequence of $2 m-1$ 3-cycles. $u_{1}, \ldots, u_{2 m+1}$ are neighbours (some may be identical) of $C$.

it would suffice to show that if $|H|>1$ then

$$
\|H\| \geq \frac{5}{3}|H|-\frac{5}{3}-\frac{n_{d}}{3} .
$$

Thus, the following sections are devoted, very roughly speaking, to studying the structure of $H(G)$ in order to prove the lower bound in (2).

The following construction shows that to prove Theorem 1.2, we only need to consider 4-critical graphs with no $>3$-cycle in their connected lowvertex subgraph.

Construction 1.8 For every $(2 m+1)$-cycle $C$ in $L(G)(m \geq 2)$ repeat the following operation. Replace $C$ with a sequence of $2 m-13$-cycles to obtain another 4-critical graph $G^{\prime}$ as shown by the example in Figure 1. Graph $G^{\prime}$ has $4 m-4$ more vertices and $6 m-6$ more edges than graph $G$. Thus, $\|G\| \geq \frac{5}{3}|G|-\frac{2}{3}$ if $\left\|G^{\prime}\right\| \geq \frac{5}{3}\left|G^{\prime}\right|-\frac{2}{3}$. The 4-criticality of $G^{\prime}$ is easy to see by observing that a 3-colouring of the rest of $G$ (or $G^{\prime}$ ) cannot be extended to $C$ (or to the sequence of 3 -cycles) iff $u_{1}, \ldots, u_{2 m+1}$ have the same colour. It is clear that this operation does not create new $>3$-cycles in the low-vertex subgraph. 


\section{Proof outline}

We begin by proving a special case of the theorem which gives the reader a taste of the complete proof.

Special Case 2.1 If $G$ is a 4-critical graph where $L(G)$ is connected and has no d-vertex then $\|G\| \geq \frac{5}{3}|G|-\frac{2}{3}$.

It suffices to prove Inequality $(2)$, which with $n_{d}=0$, states that

$$
\|H\| \geq \frac{5}{3}|H|-\frac{5}{3} .
$$

We may assume that $L \neq \emptyset$ as otherwise Inequality (2) follows from the fact that every vertex of $H$ has degree at least 4. By Corollary 1.7, we may also assume that $|H| \geq 2$. Since every 4-critical graph is 2-connected, this implies $|N(L)| \geq 2$.

The most useful implication of $G$ having no $d$-vertices is that in every colouring of $H$, all vertices in $N(L)$ have the same colour by Lemma 1.5. Among all possible 3 -colourings of $H$, we pick the one that minimizes the number of vertices with the same colour as those in $N(L)$; suppose that colour is 1 . This minimality, and the fact that $|N(L)| \geq 2$, implies that every vertex of colour 1 has at least one neighbour of each of the colours 2 and 3 .

Now, we count the number of edges in $H$ in two ways:

$$
\|H\| \geq|N(L)|+2|H \backslash N(L)| .
$$

Proof of (4): For every vertex $v \in H \backslash N(L)$, $\operatorname{deg}_{H}(v) \geq 4$, and for every vertex $v \in N(L), \operatorname{deg}_{H}(v) \geq 2$ since it has at least one neighbour of each of colours 2 and 3 . 
We next prove:

$$
\|H\| \geq 2|N(L)|+\frac{3}{2}|H \backslash N(L)|-2 .
$$

This will prove Special Case 2.1 as using the lower bounds on $\|H\|$ given by Inequalities (4) and (5), we have

$$
\|H\| \geq \frac{1}{3}(|N(L)|+2|H \backslash N(L)|)+\frac{2}{3}\left(2|N(L)|+\frac{3}{2}|H \backslash N(L)|-2\right)>\frac{5}{3}|H|-\frac{5}{3}
$$

as we required.

Proof of (5): Let $H_{1,2}$ and $H_{1,3}$ be the subgraphs of $H$ induced by the vertices of colours 1 and 2 and by the vertices of colours 1 and 3, respectively. Let $P_{2}$ (or $P_{3}$ ) be the set of vertices in $H_{1,2}$ (or $H_{1,3}$ ) that do not have any neighbour of colour 3 (or colour 2). Let $H_{N(L), 2}$ be a component of $H_{1,2}-P_{2}$ that intersects $N(L)$. If $H_{N(L), 2}$ does not contain all of $N(L)$, then by colouring all vertices in $P_{2}$ with colour 3 and swapping the colours on $H_{N(L), 2}$, we obtain a 3 -colouring of $H$ in which not all vertices of $N(L)$ have the same colour. By Lemma 1.5, this can be extended to a 3-colouring of $G$, thus contradicting the choice of $G$. So, $H_{N(L), 2}$ must contain all of $N(L)$. Similarly, let $H_{N(L), 3}$ be the component of $H_{1,3}-P_{3}$ that contains $N(L)$. Let $Q$ be the subgraph obtained by deleting all edges of $H_{N(L), 2}$ and $H_{N(L), 3}$ from $H$.

In the following argument, for $H^{\prime} \subseteq H$, let $\left(H^{\prime}\right)^{i}$ denote the set of vertices of $H^{\prime}$ of colour $i$. Then, every vertex in exactly one of $\left(H_{N(L), 2} \backslash N(L)\right)^{1}$ and $\left(H_{N(L), 3} \backslash N(L)\right)^{1}$ has degree at least 1 in $Q$ since it is adjacent to vertices of both colours 2 and 3. Also, every vertex in $\left(H_{N(L), 2}\right)^{2}$ and $\left(H_{N(L), 3}\right)^{3}$ has degree at least 1 in $Q$ since no such vertex lies in $P_{2}$ and $P_{3}$. Finally, every 
vertex in $H-\left(V\left(H_{N(L), 2}\right) \cup V\left(H_{N(L), 3}\right)\right)$ has degree at least four in $Q$. This yields

$$
\begin{aligned}
\|H\|= & \left\|H_{N(L), 2}\right\|+\left\|H_{N(L), 3}\right\|+\|Q\| \\
\geq & \left(\left|H_{N(L), 2}\right|-1\right)+\left(\left|H_{N(L), 3}\right|-1\right)+\frac{1}{2} \sum_{v \in V(Q)} \operatorname{deg}_{Q}(v) \\
\geq & \left(|N(L)|+\left|\left(H_{N(L), 2} \backslash N(L)\right)^{1}\right|+\left|\left(H_{N(L), 2}\right)^{2}\right|-1\right)+ \\
& \left(|N(L)|+\left|\left(H_{N(L), 3} \backslash N(L)\right)^{1}\right|+\left|\left(H_{N(L), 3}\right)^{3}\right|-1\right)+ \\
& \frac{1}{2}\left(\left|\left(H_{N(L), 2} \backslash N(L)\right)^{1} \triangle\left(H_{N(L), 3} \backslash N(L)\right)^{1}\right|\right. \\
& \left.+\left|\left(H_{N(L), 2}\right)^{2}\right|+\left|\left(H_{N(L), 3}\right)^{3}\right|+4\left|H \backslash\left(V\left(H_{N(L), 2}\right) \cup V\left(H_{N(L), 3}\right)\right)\right|\right) \\
\geq & 2|N(L)|+\frac{3}{2}\left|\left(H_{N(L), 2} \cup H_{N(L), 3}\right) \backslash N(L)\right|-2+4\left|H-\left(V\left(H_{N(L), 2}\right) \cup V\left(H_{N(L), 3}\right)\right)\right| \\
\geq & 2|N(L)|+\frac{3}{2}|H \backslash N(L)|-2
\end{aligned}
$$

as required.

Remark 2.2 It is valuable to note that the -2 in (5) corresponds to the number of components of $H_{1,2}-P_{2}$ and $H_{1,3}-P_{3}$ that contain all of $N(L)$.

The proof of Inequality (2), basically, follows the same idea; however, a naive extension of the above proof will not work. The main difficulty is that, in the presence of $d$-vertices, vertices in $N(L)$ are not necessarily of the same colour. This has a few repercussions: $N(L)$ may intersect many different components of $H_{1,2}$ and $H_{1,3}$ (and $H_{2,3}$ which will be defined similarly). It is not very hard to show that the number of components of $H_{1,2}, H_{1,3}$ and $H_{2,3}$ that intersect $N(L)$ is at most $n_{d}+2$ and this is sharp. Thus, recalling Remark 2.2, with a bit more work, this allows us to replace (5) by

$$
\|H\| \geq 2|N(L)|+\frac{3}{2}|H \backslash N(L)|-\left(n_{d}+2\right) .
$$


But this, with (4), is not enough to prove Inequality (2). So, we have to focus more closely on the degrees in $H$ of the vertices in $N(L)$. Let $\alpha$, $\beta$ and $\gamma$ denote the number of vertices in $N(L)$ with at least three, exactly two and exactly one neighbour(s) in $H$, respectively. So, (4) becomes:

$$
\|H\| \geq 2|H \backslash N(L)|+\frac{3}{2} \alpha+\beta+\frac{1}{2} \gamma .
$$

Unfortunately, (6) and (7) are not enough to yield (3), which we need. Roughly speaking (see below), by being much more careful, we can improve (6) to:

$$
\|H\| \geq 2|N(L)|+\frac{3}{2}|H \backslash N(L)|-\left(n_{d}-\beta-2 \gamma+5\right) .
$$

Inequalities (7) and (8) imply Inequality (2) and hence our main theorem, as follows:

$$
\begin{aligned}
\|H\| & \geq \frac{2}{3}\left(2|H \backslash N(L)|+\frac{3}{2} \alpha+\beta+\frac{1}{2} \gamma\right)+\frac{1}{3}\left(2|N(L)|-\left(n_{d}-\beta-2 \gamma+5\right)+\frac{3}{2}|H \backslash N(L)|\right) \\
& \geq \frac{11}{6}|H \backslash N(L)|+\frac{2}{3}|N(L)|+(\alpha+\beta+\gamma)-\frac{1}{3} n_{d}-\frac{5}{3} \\
& \geq \frac{5}{3}(|N(L)|+|H \backslash N(L)|)-\frac{n_{d}}{3}-\frac{5}{3} \\
& \geq \frac{5}{3}|H|-\frac{n_{d}}{3}-\frac{5}{3}
\end{aligned}
$$

Actually, Inequality (8) does not hold for every 4-critical graph. Therefore, for technical reasons, we work with pruned L-critical graphs - a class of graphs which we define in Section 4. Lemmas 5.2 and 5.3, the analogues of Inequalities (7) and (8), show that Inequality (2) holds for pruned $L$ critical graphs which satisfy specific degree constraints, and thus implying Theorem 1.2 for that class of graphs. In Section 4, we prove that if Theorem 1.2 holds for such graphs then it holds for every 4-critical graph whose low-vertex subgraph is connected (see Lemma 4.2 and Construction 4.3). 


\section{A generalized structure}

Our proof of Lemma 5.1, the main ingredient for Lemma 5.3, is by induction. When carrying out our induction, it is problematic to show that the graphs we reduce to remain 4-critical. We overcome this problem by extending our setting to a broader class of graphs, which we call $L$-critical.

Let $L$ be a 4 -Gallai tree, and let $F$ be a graph that contains $L$ as an induced subgraph such that $\operatorname{deg}_{F}(v)=3$ for every vertex $v \in V(L)$. We say $F$ is $L$-critical if $\chi(F)=4$ and $\chi(F-L) \leq 3$. Thus, a 4-critical graph $G$ with a connected low-vertex subgraph is $L(G)$-critical. Define $H(F)$, or $H$ when there is no ambiguity, to be the graph $F-L . F$ is not necessarily 4-critical, and in particular, possibly some vertices of $H$ have degree $0,1,2$ or 3 .

For any Gallai tree $L$ and any $L$-critical graph $F$, we have the following two observations.

Observation 3.1 If $L^{\prime} \subseteq L$ is connected, then for any vertex $v \in L^{\prime}$, any 3-colouring of $F-L^{\prime}$ has an extension to $L^{\prime}-v$.

Observation 3.2 If $u \in N(L)$ then $\operatorname{deg}_{F}(u) \geq 3$. Moreover, if $u \in N(L)$ and $\operatorname{deg}_{F}(u)=3$, then $L \cup\{u\}$ is also a 4-Gallai tree, and $F$ is $(L \cup\{u\})$ critical.

For an $L$-critical graph $F$, define $\|L, H\|, d$-vertices and $n_{d}$ analogously to the definitions in Section 1. The proofs of Lemma 3.3 and Corollary 3.4 below are exactly the same as the proofs of Lemma 1.6 and Corollary 1.7.

Lemma $3.3\|L\|+\|L, H\| \geq \frac{5}{3}|L|+1+\frac{n_{d}}{3}$. 
Corollary 3.4 If $\|H\| \leq 1$ then $\|F\| \geq \frac{5}{3}|F|-\frac{2}{3}$.

Let $\phi$ be a 3-colouring of $H$. Let $P_{\phi}$ consist of every vertex $v \in H \backslash N(L)$ such that only one colour appears in $N_{H}(v)$. Roughly speaking, $P_{\phi}$ corresponds to vertices in $P_{2}$ and $P_{3}$ in Section 2. For every vertex $v \in P_{\phi}$, there is an available colour (other than its own colour) that can be assigned to $v$ without violating the properness of the 3 -colouring. In fact, we can recolour any independent set of $P_{\phi}$, without violating the properness of the 3-colouring. However, when we recolour a vertex $v \in P_{\phi}$, graph $H$ obtains a new colouring, say $\phi^{\prime}$, and thus $P_{\phi^{\prime}}$ can be different from $P_{\phi}$.

We will need to focus on possible ways to recolour the vertices of $N(L)$. One way is to do so using traditional kempe chains. But such switches will not suffice for our purposes. Instead we need to consider a combination of recolouring an independent set of $P_{\phi}$ and then switching on a kempe chain. In what follows, we will define sets of vertices in $N(L)$ whose colours can be switched in this manner. We will denote such sets by kempes.

Let $I$ be a maximal independent subset of $P_{\phi}$. Define $H_{1,2}$ to be the set of the components of the subgraph of $H \backslash I$ induced by the vertices of colours 1 or 2 that intersect $N(L)$. The set of vertices of $N(L)$ in a particular component of $H_{1,2}$ is called a $(1,2)$-kempe. Let $\Omega_{1,2}$ denote the set of $(1,2)$ kempes. More formally, let $\Omega_{1,2}=\left\{h \cap N(L): h\right.$ is a component in $\left.H_{1,2}\right\}$. Define (1,3)-kempes, $(2,3)$-kempes, $\Omega_{1,3}$ and $\Omega_{2,3}$, similarly. Then, $\Omega=$ $\left(\Omega_{1,2}, \Omega_{1,3}, \Omega_{2,3}\right)$ is called a valid collection of kempes for $\phi$. A kempe is an $(i, j)$-kempe for any $1 \leq i<j \leq 3$. Thus, every vertex in $N(L)$ belongs to two kempes; e.g., a vertex in $N(L)$ of colour 1 belong to one $(1,2)$-kempe and one $(1,3)$-kempe. 
Note that for an $L$-critical graph $F$, we may have many different collection of kempes depending on our choice of the 3-colouring $\phi$ and the set $I$. From the 3-colouring $\phi$ of $H$ we can obtain another 3 -colouring of $H$, say $\phi^{\prime}$, by performing a switch on a kempe $\omega$ as follows.

Suppose that $\omega \in \Omega_{1,2}$ where $\omega=Q \cap N(L)$ and $Q$ is a component of $H_{1,2}(\phi)$. We would like to obtain a new 3 -colouring of $H$ in which every vertex in $N(L)$, except for those in $\omega$, still has its original colour, and the vertices in $\omega$ have swapped their colours. This can be done as follows.

Switch: For every vertex $v \in I \cap N(Q)$ : if the colour of $v$ is not 3 , then it can be switched to 3 ; make that switch. Now, every vertex in $N(Q)$ has colour 3. Then, we switch the colours in $Q$, i.e. recolour the vertices of colour 1 with colour 2 and those of colour 2 with colour 1, without violating the properness of the 3 -colouring of $H$.

Note that it is possible that $\omega \subseteq N(L)$ represents two different kempes; e.g. a (1,2)-kempe and a (1,3)-kempe (in this case, every vertex in $\omega$ must be of colour 1). Then, there are two possible switches that can be performed on $\omega$.

\section{Pruned critical graphs and credits}

As we said in Section 2, the proof of the inequality in Lemma 5.3, the analogue of Inequality (8), involves some technicality: (i) obtaining $n_{d}-$ $\beta-2 \gamma+5$, the analogue of the -2 term in Remark 2.2, requires working with pruned $L$-critical graphs - a subclass of $L$-critical graphs that we define

below; (ii) obtaining the coefficients $\frac{3}{2}$ and $\frac{5}{3}$ requires, roughly speaking, 
specific degree constraints on vertices of $H$.

Suppose that $L$ is a 4 -Gallai tree and $F$ is an $L$-critical graph. A connected subgraph $W$ of $L$ induced by a subset of $d$-vertices is called a wing if the subgraph has at most one neighbour in $L-W$. We observe that wings have no cycles. If vertex $v$ of a wing $W$ is adjacent to a vertex in $L-W$ then $v$ is called the root of $W$. A wing $W$ is called pendant if the root of $W$ is adjacent to a cycle in $L-W$.

Next, we extend the definition of $\alpha, \beta, \gamma$ from Section 2 to this setting. Let $N_{\alpha}$ denote the subset of $N(L)$ with exactly one neighbour in $L$, let $N_{\beta}$ denote the subset of $N(L)$ with at least two neighbours in $L$ and at least two neighbours in $F-L$, and let $N_{\gamma}$ denote the subset of $N(L)$ with at least two neighbours in $L$ and at most one neighbour in $F-L$. Again, we emphasize that in an $L$-critical graph, vertices of $F-L$ may have degree $<4$. So $\left\{N_{\alpha}, N_{\beta}, N_{\gamma}\right\}$ is a partition of $N(L)$. Let $\alpha=\left|N_{\alpha}\right|, \beta=\left|N_{\beta}\right|$ and $\gamma=\left|N_{\gamma}\right|$

Lemma 4.1 Suppose that $v \in N_{\gamma}$, and that $W$ is a wing in L. If $v$ has exactly three neighbours in $L$, then at least one of them is in $L-W$.

Proof. Assume otherwise, and let $x_{1}, x_{2}$ and $x_{3}$ be the three neighbours of $v$ in $W$. Let $y \in W$ be the common vertex among the three paths in $R$ that join pairs of $x_{1}, x_{2}$ and $x_{3}$, and let $R$ be the subgraph consisting of the three disjoint paths from $v$ to $y$ in $W \cup\{v\}$. By Observation 3.1, we can consider a 3 -colouring of $F-R$. Since $v \in N_{\gamma}, \operatorname{deg}_{H}(v) \leq 1$. Also, for all $u \in R \backslash v, \operatorname{deg}_{F}(u)=3$. Thus, every vertex of $R$ has at most one coloured neighbour, and moreover, $y$ has no coloured neighbours. A simple 
case analysis shows that the 3 -colouring can be extended to $R$ (details of this case analysis appear in [Far05]). Contradiction.

Definition 1 Suppose that $L$ is a 4-Gallai tree. An L-critical graph $F$ is called pruned if (i) $L$ is a 3-cycle and $|N(L)|=1$ or (ii) $F$ has the following properties:

(P1) L has no >3-cycle.

(P2) No vertex in $H$ of degree 3 has exactly two neighbours in $L$.

(P3) Every vertex in $H$ that is adjacent to a cycle in $L$ belongs to $N_{\alpha}$.

(P4) Every pendant wing $W$ is adjacent to a vertex in $N_{\alpha}$.

\subsection{Pruning a critical graph}

As we explained at the beginning of Section 4, we also require some degree constraints on the vertices of a pruned $L$-critical graph. Instead of considering a simple edge-count, it will be convenient to consider a more general notion: We will define the credit of vertex $v$ which we denote by $\operatorname{credit}(v)$ to be a nonnegative real value. The credit of a graph $G$ is defined to be $\|G\|+\sum_{v \in V(G)} \operatorname{credit}(v)$. If $L$ is a 4 -Gallai tree and $F$ is an $L$-critical graph, then $F$ meets degree criteria if for every vertex $v \in H(F)$ :

(Q0) If $v \notin N(L)$ then $\frac{\operatorname{deg}_{F}(v)}{2}+\operatorname{credit}(v) \geq \frac{5}{3}$;

(Q1) If $v \notin N(L)$ then $\operatorname{deg}_{F}(v) \geq 3$;

(Q2) If $v \in N(L)$ then $\operatorname{deg}_{F}(v) \geq 4$.

Lemma 4.2 If $G$ is a 4-critical graph where $L(G)$ is connected and $|H(G)|>$ 1, then we can form a new 4-Gallai tree $L$ and a pruned $L$-critical graph $F$ that meets degree criteria such that: 
(R) If $\operatorname{credit}(F) \geq \frac{5}{3}|F|-\frac{2}{3}$ then $\|G\| \geq \frac{5}{3}|G|-\frac{2}{3}$.

This will be shown by the following construction.

Construction 4.3 We present the following operations ZQ1, ZQ2, ZP1, ZP3, and ZP4 which can be applied if properties (Q1), (Q2), (P1), (P3), and (P4), respectively, do not hold. These operations are listed in order of their priorities. Thus, e.g. if we perform operation ZP3, then we must not be able to carry out ZQ1, ZQ2 or ZP1, and so we know that the graph satisfies properties (Q1), (Q2) and (P1). Notice that (Q2) implies (P2), and so we do not have an operation specifically for (P2).

Performing these operations does not necessarily reduce the number of violations of our properties. While eliminating one violation, we might introduce others. However, after every operation either (i) $|H|$ decreases, or (ii) $|H|$ does not change but the number of $>3$-cycles in $L$ decreases, or (iii) $|H|$ does not change but $|L|$ decreases and the number of $>3$-cycles in $L$ does not increase. So, after finitely many operations, we will reach a state where none of them can be applied. Then the resulting graph will be pruned and will meet degree criteria.

We begin with $F=G$ and $L=L(G)$, and note that trivially, (Q0) and $(\mathrm{R})$ both hold for this graph. We show that every operation preserves properties (Q0) and (R). This allows us to assume that (Q0) and (R) always hold when we carry out our operations. In what follows, we use $L$ and $F$ to denote the 4-Gallai tree and the $L$-critical graph before an operation, and $L^{\prime}$ and $F^{\prime}$ to denote the new 4 -Gallai tree and the new $L^{\prime}$-critical graph after the operation takes place. Therefore, in order to show that $F^{\prime}$ satisfies $(\mathrm{R})$, it 
would suffice to show that if $\operatorname{credit}\left(F^{\prime}\right) \geq \frac{5}{3}\left|F^{\prime}\right|-\frac{2}{3}$ then $\operatorname{credit}(F) \geq \frac{5}{3}|F|-\frac{2}{3}$, since $F$ satisfies $(\mathrm{R})$.

Operation ZQ1. If for a vertex $v \notin N(L), \operatorname{deg}_{F}(v) \leq 2$ then obtain $F^{\prime}$ from $F$ as follows (i) delete vertex $v$ from $F$, and (ii) for each $u \in N(v) \backslash N(L)$ give $\frac{1}{6}$ credit to $u$ if $\operatorname{deg}_{F^{\prime}}(u)=3$ and $\frac{1}{2}$ if $\operatorname{deg}_{F^{\prime}}(u) \leq 2$. In this operation, $L^{\prime}$ remains the same as $L$, and clearly $F^{\prime}$ is $L^{\prime}$-critical. Neighbours of $v$ are the only vertices whose degree and credit are affected by this operation; it is easy to see that since these vertices satisfied (Q0) in $F$, they satisfy (Q0) in $F^{\prime}$. For $(\mathrm{R})$, since $\operatorname{credit}(v) \geq\left(\frac{5}{3}-\frac{\operatorname{deg}(v)}{2}\right)$, if $\operatorname{credit}\left(F^{\prime}\right) \geq \frac{5}{3}\left|F^{\prime}\right|-\frac{2}{3}$ then

$$
\begin{aligned}
\operatorname{credit}(F) & \geq \operatorname{credit}\left(F^{\prime}\right)+\operatorname{deg}(v)+\operatorname{credit}(v)-\frac{\operatorname{deg}(v)}{2} \\
& \geq \frac{5}{3}(|F|-1)-\frac{2}{3}+\frac{5}{3}=\frac{5}{3}|F|-\frac{2}{3} .
\end{aligned}
$$

Remark 4.4 Note that the above inequality is tight only if we give $\frac{1}{2}$ edge credit to every neighbour of $v$, i.e. if all neighbours of $v$ have no neighbour in $L$ and are of degree $\leq 3$. Otherwise, we could give at least $\frac{1}{3}$ edge credit to another vertex in $F^{\prime}$ without violating $(\mathrm{R})$. We need this observation later in Operation ZP4.

Operation ZQ2. If $\operatorname{deg}_{F}(v) \leq 3$ for a vertex $v \in N(L)$, then by Observation $3.2, \operatorname{deg}_{F}(v)=3$ since $F$ is $L$-critical. We move vertex $v$ to $L$, i.e. $L^{\prime}=L \cup\{v\}$. In this operation, $F^{\prime}$ remains the same as $F$. By Observation $3.2, L^{\prime}$ is a 4 -Gallai tree, and $F$ is $L^{\prime}$-critical. Also, the degree and the credit of no vertex in $F$ is changed. So, $F^{\prime}$ satisfies (Q0) and (R).

Operation ZP1. If $L$ has a $>3$-cycle, we perform Construction 1.8 in which $L^{\prime}$ and $F^{\prime}$ are obtained from $L$ and $F$ by replacing the $>3$-cycle by 
a sequence of 3 -cycles. $F^{\prime}$ is easily seen to be $L^{\prime}$-critical iff $F$ is $L$-critical. Moreover, the degree and the credit of no vertex in $H$ was changed, and so $F^{\prime}$ satisfies (Q0). Also, since the edge credit of no vertex was changed, the argument in Construction 1.8 shows that $F^{\prime}$ satisfies (R).

Operation ZP3. If a vertex $v \in H(F)$ is adjacent to at least two vertices of $L$ then at least one of them is in a 3-cycle $C$ in $L$ as by the priorities of the operations, $F$ satisfies (P1). Let $u_{1}$ and $u_{2}$ be the other two neighbours of $C$. Now, $L^{\prime}$ (or $F^{\prime}$ ) is the graph spanned by the vertices of $L$ (or $F$ ) without the vertices of cycle $C$ and adding the edge $\left(u_{1}, u_{2}\right)$. It is easy to see that $L^{\prime}$ is a 4 -Gallai tree and that $F^{\prime}$ is $L^{\prime}$-critical. In particular, if $\chi\left(F^{\prime}\right) \leq 3$, then $F^{\prime}$ has a 3 -colouring in which $u_{1}$ and $u_{2}$ must have different colours. Thus, $F-C$ has a 3 -colouring in which $u_{1}$ and $u_{2}$ have different colours. So, cycle $C$ can be coloured accordingly. Contradiction. It is also easy to see that $F^{\prime}$ satisfies (Q0) (notice that $\operatorname{deg}_{L^{\prime}}(v) \geq 1$ ). For $(\mathrm{R})$, if $\operatorname{credit}\left(F^{\prime}\right) \geq \frac{5}{3}\left|V\left(F^{\prime}\right)\right|-\frac{2}{3}$ then

$$
\operatorname{credit}(F) \geq \operatorname{credit}\left(F^{\prime}\right)+5 \geq \frac{5}{3}(|F|-3)-\frac{2}{3}+5=\frac{5}{3}|F|-\frac{2}{3} .
$$

Operation ZP4. Suppose that there is a pendant wing $W$ that has no neighbour in $N_{\alpha}$. Suppose that $u$ is the root of $W$ and that $u$ is adjacent to a cycle $C$ in $L$, and let $u_{1}$ and $u_{2}$ be the other two neighbours of $C$. Operation ZP4 consists of at most two steps.

Step 1. Let $L^{\prime}$ (or $F^{\prime}$ ) be the graph obtained by removing the vertices of wing $W$ and cycle $C$ from $L$ (or $F$ ) and adding the edge $\left(u_{1}, u_{2}\right)$. Now, if a vertex $v$ had a neighbour in $L$ but has no neighbour in $L^{\prime}$, then we give $\frac{1}{6}$, $\frac{2}{3}$, or $1 \frac{1}{6}$ credit to $v$ if it becomes a vertex of degree 3,2 , or 1 , respectively. 
Therefore, $F^{\prime}$ satisfies (Q0). (Later on we see that $v$ cannot become a vertex of degree 0.)

Claim 4.5 In Step 1, the total credit given to vertices is at most $\frac{|W|+1}{3}$.

Proof. By (Q2), suppose that for every edge between $W$ and $H$ we give at most $\frac{1}{3}$ credit to the endpoint in $H$ (or $\frac{1}{6}$ credit if it becomes a vertex of degree 3) unless it has a neighbour in $L^{\prime}$. So, if a vertex in $N_{\beta}$ or $N_{\gamma}$ has no neighbour in $L^{\prime}$, then it has at least two or at least four neighbours in $W$ (by Lemma 4.1), and it gets at least $\frac{2}{3}$ or $1 \frac{1}{3}$ credit, respectively, as required in any operation. Since there are $|W|+1$ edges between $W$ and $H$, at most $\frac{|W|+1}{3}$ credit is given to vertices.

It is easy to see that $L^{\prime}$ is a 4 -Gallai tree and that $F^{\prime}$ is $L^{\prime}$-critical. In particular, if $\chi\left(F^{\prime}\right) \leq 3$, then $F^{\prime}$ has a 3-colouring in which $u_{1}$ and $u_{2}$ must have different colours. Thus, $F-\{C, W\}$ has a 3 -colouring in which $u_{1}$ and $u_{2}$ have different colours. By Observation 3.1, the colouring can be extended to $W$. Now, since $u_{1}$ and $u_{2}$ have different colours, the colouring can be extended to $C$. Contradiction.

The argument in Claim 4.5 implies that in the following Case 1 and Case 2, at most $\frac{|W|}{3}$ credit is given to the vertices. Operation ZP4 ends after Step 1 in these two cases.

Case 1: At least one vertex in $N_{H}(W)$ has a neighbour in $L^{\prime}$ or remains a vertex of degree $\geq 4$.

Case 2: At least two vertices of $N_{H}(W)$ have no neighbour in $L^{\prime}$ and have degree $\geq 3$. 
Now, it is easy to see that $F^{\prime}$ satisfies $(\mathrm{R})$ : If $\operatorname{credit}\left(F^{\prime}\right) \geq \frac{5}{3}\left|F^{\prime}\right|-\frac{2}{3}$ then

$$
\begin{aligned}
\operatorname{credit}(F) & =\operatorname{credit}\left(F^{\prime}\right)+2|W|+5-\frac{|W|}{3} \\
& \geq \frac{5}{3}(|F|-|W|-3)-\frac{2}{3}+\frac{5|W|}{3}+5=\frac{5}{3}|F|-\frac{2}{3} .
\end{aligned}
$$

Case 3: Neither Case 1 nor Case 2 holds.

Consider an extension of a 3 -colouring of $H$ to $L-C$. Since $\chi(F)=4$, vertices $u, u_{1}$ and $u_{2}$ must get the same colour, say 1 . Let $F(u, 2)$ denote the component of $F-C$ on vertices of colours 1 and 2 that contains vertex $u$. Recolour vertices of colours 1 and 2 in $F(u, 2)$ with colours 2 and 1 , respectively. Now, vertex $u$ has colour 2 , and consequently, the colour of vertices $u_{1}$ and $u_{2}$ must have been changed to colour 2 , as otherwise $C$ can be coloured. This implies that $F(u, 2)$ contains both vertices $u_{1}$ and $u_{2}$. Thus, by the tree structure of wing $W$, there is a vertex $v_{1} \in F(u, 2) \cap N_{H}(W)$ that is connected via a path $p_{1} \subseteq F(u, 2)$ to a vertex in $H$ that has a neighbour in $L-W$. By considering $F(u, 3)$, which is defined similarly, the same argument proves that there exists a vertex $v_{2} \in F(u, 3) \cap N_{H}(W)$ (notice that possibly $\left.v_{1}=v_{2}\right)$ that is connected via a path $p_{2} \subseteq F(u, 3)$ to a vertex in $H$ that has a neighbour in $L-W$. All vertices in $p_{1}$ have colours 1 and 2 and all vertices in $p_{2}$ have colours 1 and 3 , and so, $p_{1}$ and $p_{2}$ are edge disjoint. Let $v_{i}^{\prime}$ be the closest vertex on $p_{i}$ to $v_{i}$ that has degree $\geq 4$. Such a vertex exists since $p_{i}$ connects $v_{i}$ to a vertex that has a neighbour in $L-W$, and such a vertex has degree $\geq 4$ by $(\mathrm{Q} 2)$ and the fact that Case 1 does not hold. Since Case 2 does not hold, at least one of $v_{1}$ and $v_{2}$, say $v_{1}$, becomes a vertex of degree $\leq 2$ with no neighbour in $L^{\prime}$. Let $p_{1}=\left(v_{1}=v_{1}^{1}, v_{2}^{1}, \ldots, v_{k}^{1}, v_{1}^{\prime}, \ldots\right)$. Notice that if $v_{1}=v_{2}$, then $\operatorname{deg}_{H}\left(v_{1}\right) \geq 4$, and so Case 1 holds. Let $v_{k^{\prime}}^{1}$ be 
the closest vertex to $v_{1}$ on $p_{1}$ that is adjacent to $v_{1}^{\prime}$. So, $k^{\prime} \leq k$.

Then we carry out Step 2 which calls Operation ZQ1 as a subroutine.

Step 2. We will apply Operation ZQ1 on vertices $v_{1}^{1}, v_{2}^{1}, \ldots, v_{k^{\prime}}^{1}$ in that order. Since $v_{1}^{\prime}$ is the first vertex along the path to have degree $\geq 4, v_{i}^{1}$ becomes a vertex of degree $\leq 2$ after the deletion of vertex $v_{i-1}^{1}$, and so we can apply Operation ZQ1 each time. When we reach $v_{k^{\prime}}^{1}$, vertex $v_{1}^{\prime}$ still has degree $\geq 4$ by the choice of $v_{k^{\prime}}^{1}$. So, when we apply Operation ZQ1 on vertex $v_{k^{\prime}}^{1}$, by Remark 4.4, we will get an extra $\frac{1}{3}$ credit. We use this extra $\frac{1}{3}$ credit to balance out the credit that we spend in Step 1.

In the following argument, $L^{\prime}$ and $F^{\prime}$ denote the 4-Gallai tree and the $L^{\prime}$-critical graph after Step 1 , and $L^{\prime \prime}$ and $F^{\prime \prime}$ denote the 4-Gallai tree and the $L^{\prime \prime}$-critical graph after Step 2. After Step 2, by the proof of Operation ZQ1, it is clear that $L^{\prime \prime}$ is a 4 -Gallai tree, $F^{\prime \prime}$ is $L^{\prime \prime}$-critical, and $F^{\prime \prime}$ satisfies (Q0).

Thus, it remains to prove that $F^{\prime \prime}$ satisfies (R). If $\operatorname{credit}\left(F^{\prime \prime}\right) \geq \frac{5}{3}\left|F^{\prime \prime}\right|-\frac{2}{3}$ then

$\operatorname{credit}\left(F^{\prime}\right) \geq \operatorname{credit}\left(F^{\prime \prime}\right)-\frac{5 k}{3}-\frac{1}{3} \geq \frac{5}{3}\left(\left|F^{\prime}\right|-k\right)-\frac{2}{3}-\frac{5 k}{3}+\frac{1}{3}=\frac{5}{3}\left|F^{\prime}\right|-\frac{1}{3}$.

By Claim 4.5, in Step 1, we give at most $\frac{|W|+1}{3}$ credit to the vertices. If $\operatorname{credit}\left(F^{\prime}\right) \geq \frac{5}{3}\left|F^{\prime}\right|-\frac{1}{3}$ then

$$
\begin{aligned}
\operatorname{credit}(F) & \geq\left(\operatorname{credit}\left(F^{\prime}\right)+2|W|+5-\frac{|W|+1}{3}\right)-\frac{5 k}{3} \\
& \geq \frac{5}{3}(|F|-|W|-3)-\frac{1}{3}+\frac{5|W|}{3}-\frac{1}{3}+5=\frac{5}{3}|F|-\frac{2}{3} .
\end{aligned}
$$

Thus, if $\operatorname{credit}\left(F^{\prime \prime}\right) \geq \frac{5}{3}\left|F^{\prime \prime}\right|-\frac{2}{3}$ then $\operatorname{credit}(F) \geq \frac{5}{3}|F|-\frac{2}{3}$. 


\section{The formal versions of Inequalities (7) and (8)}

For a valid collection of kempes $\Omega=\left(\Omega_{1,2}, \Omega_{1,3}, \Omega_{2,3}\right)$ define $|\Omega|=\left|\Omega_{1,2}\right|+$ $\left|\Omega_{1,3}\right|+\left|\Omega_{2,3}\right|$. Recall the definitions of $n_{d}, \beta$ and $\gamma$ from Section 3. We provide the proof of the following key lemma in the next subsection.

Lemma 5.1 Let $F$ be a pruned L-critical graph for a 4-Gallai tree L. Let $\phi$ be a 3-colouring of $H$, and let $\Omega=\left(\Omega_{1,2}, \Omega_{1,3}, \Omega_{2,3}\right)$ be a valid collection of kempes for $\phi$. Then $|\Omega| \leq n_{d}-\beta-2 \gamma+5$.

Define $H_{+}=\{v \in H \backslash N(L): \operatorname{credit}(v)>0\}$. We are now ready to state our formal versions of Inequalities (7) and (8).

Lemma 5.2 If $F$ is a pruned L-critical graph that meets the degree criteria, then $\operatorname{credit}(H) \geq 2\left|H \backslash N(L)-H_{+}\right|+\frac{5}{3}\left|H_{+}\right|+\frac{3}{2} \alpha+\beta+\frac{1}{2} \gamma$.

Proof. Since $F$ meets the degree criteria, if $v \in H(F)$ and $\operatorname{credit}(v)=0$ then $\operatorname{deg}(v) \geq 4$. As $\operatorname{credit}(H)=\sum_{v \in H}\left(\frac{\operatorname{deg}_{H}(v)}{2}+\operatorname{credit}(v)\right)$, recalling the definitions of $\alpha, \beta$ and $\gamma$ and applying (Q4), we only need to show that if $v \in N_{\gamma}$ then $\operatorname{deg}_{H}(v) \neq 0$. Assume otherwise and consider a 3-colouring of $H$. Let $R$ be the minimal connected induced subgraph of $L$ that spans $N_{L}(v)$. By (P3), $v$ is not adjacent to any vertices of 3 -cycles in $L$, and so $R$ has a vertex $y$ with $\operatorname{deg}_{R}(y)=1$ that is a neighbour of $v$. Let $z$ be the other neighbour of $y$ outside of $R$. Consider any 3 -colouring of $F-R$. Since $v$ has no neighbour in $F-R$, we can change the colour of $v$ to the colour of $z$. Now, $y$ has two neighbours of the same colour. So we can extend the colouring to $R$ by colouring its vertices one-at-a-time, ending with $y$. Contradiction. 
Lemma 5.3 If $F$ is a pruned L-critical graph that meets the degree criteria, then $\operatorname{credit}(H) \geq 2|N(L)|-\left(n_{d}-\beta-2 \gamma+5\right)+\frac{3}{2}\left|H \backslash N(L)-H_{+}\right|+\frac{5}{3}\left|H_{+}\right|$.

Proof. Let $\phi$ be a 3-colouring of $H$ and $I$ be a maximal independent set of $P_{\phi}$. Lemma 5.1 implies that a valid collection of kempes has at most $n_{d}-\beta-$ $2 \gamma+5$ kempes. Let $H_{1,2}$ be those components of $H \backslash I$ on vertices of colours 1 and 2 that intersect $N(L)$. Define $H_{1,3}$ and $H_{2,3}$ similarly. Recall that there is a one-to-one correspondence between the components of $H_{1,2}, H_{1,3}$ and $H_{2,3}$ and the kempes. Also, let $Q$ be the subgraph obtained by deleting all edges of $H_{1,2}, H_{1,3}$ and $H_{2,3}$ from $H$. $\operatorname{credit}(H)=\left\|H_{1,2} \cup H_{1,3} \cup H_{2,3}\right\|+\|Q\|+$ $\sum_{v \in V(H)} \operatorname{credit}(v)$. Let $T_{0}, T_{1}$ and $T_{2}$ be the set of vertices in $H \backslash N(L)$ that are in none, one and two components of $H_{1,2}, H_{1,3}$ and $H_{2,3}$, respectively. So, ||$H_{1,2} \cup H_{1,3} \cup H_{2,3} \| \geq 2|N(L)|+\left|T_{1}\right|+2\left|T_{2}\right|-\left(n_{d}-\beta-2 \gamma+5\right)$ as every vertex in $N(L)$ appears in exactly two kempes, and thus, in exactly two components. Every vertex in $T_{1}$ has an edge to a vertex in $I$ or has an edge to a vertex of the third colour. Furthermore, $\sum_{v \in V(H)} \operatorname{credit}(v) \geq \frac{2}{3}\left|H_{+}\right|$. Therefore, $\|Q\|=\frac{1}{2} \sum_{v \in V(H)} \operatorname{deg}_{Q}(v) \geq \frac{1}{2}\left(4\left|T_{0}-H_{+}\right|+\left|T_{1}-H_{+}\right|+2\left|T_{+}-T_{0}\right|\right)$. Thus, $\operatorname{credit}(H) \geq 2|N(L)|-\left(n_{d}-\beta-2 \gamma+5\right)+\frac{3}{2}\left|H \backslash N(L)-H_{+}\right|+\frac{5}{3}\left|H_{+}\right|$.

Proof of Theorem 1.2 By Lemma 4.2, it would suffice to prove that for any 4-Gallai tree $L$, if $F$ is a pruned $L$-critical graph that meets the degree criteria, then $\operatorname{credit}(F) \geq \frac{5}{3}|F|-\frac{2}{3}$. We will use an argument like the one at the end of Section 2: By Lemmas 5.2 and 5.3,

$$
\begin{aligned}
\operatorname{credit}(H) \geq & \frac{2}{3}\left(2\left|H \backslash\left(N(L) \cup H_{+}\right)\right|+\frac{5}{3}\left|H_{+}\right|+\frac{3}{2} \alpha+\beta+\frac{1}{2} \gamma\right) \\
& +\frac{1}{3}\left(2|N(L)|-\left(n_{d}-\beta-2 \gamma+5\right)+\frac{3}{2}\left|H \backslash\left(N(L) \cup H_{+}\right)\right|+\frac{5}{3}\left|H_{+}\right|\right) \\
\geq & \frac{11}{6}\left|H \backslash\left(N(L) \cup H_{+}\right)\right|+\frac{5}{3}\left|H_{+}\right|+\frac{5}{3}|N(L)|-\frac{n_{d}}{3}-\frac{5}{3}
\end{aligned}
$$




$$
\geq \frac{5}{3}|H|-\frac{n_{d}}{3}-\frac{5}{3}
$$

Now, by Lemma $3.3, \operatorname{credit}(F)=\|L\|+\|L, H\|+\operatorname{credit}(H) \geq \frac{5}{3}|F|-\frac{2}{3}$, as we required.

\subsection{Proof of Lemma 5.1}

First, we review some properties of $L$-critical graphs and their valid collections of kempes. Let $L$ be a 4 -Gallai tree, $F$ be an $L$-critical graph, and $\phi$ be a 3-colouring of $H$. Suppose that $\Omega=\left(\Omega_{1,2}, \Omega_{1,3}, \Omega_{2,3}\right)$ is a valid collection of kempes for $\phi$.

Let $C$ be a cycle in $L$, and let $N$ be the neighbours in $H$ of cycle $C$. From Lemma 1.5, we know that in any 3-colouring of $H$, including $\phi$ and those obtained by performing a switch on $\phi$, all vertices in $N$ have the same colour. Thus,

Observation 5.4 All vertices in $N$ belong to the same kempes of $\Omega$. Moreover, if $L-C \neq \emptyset$ and no vertex in $N$ is adjacent to a vertex in $L-C$, then $N$ is not a kempe.

Now, suppose that $v$ is a $d$-vertex with two neighbours $u_{1}$ and $u_{2}$ in $H$. In any 3-colouring of $F \backslash v$, including the extension of $\phi$ to $L \backslash v$ and those

obtained by performing a switch on $\phi$ and then extending to $L \backslash v$, vertices $u_{1}$ and $u_{2}$ must have different colours - otherwise there would be a colour available for $v$ and hence a 3 -colouring of $F$. Thus,

Observation 5.5 Let $c_{1}$ and $c_{2}$ be the colours of $u_{1}$ and $u_{2}$, respectively. Then, $u_{1}$ and $u_{2}$ belong to the same kempe of $\Omega_{c_{1}, c_{2}}$. Moreover, neither $\left\{u_{1}\right\}$ nor $\left\{u_{2}\right\}$ is a kempe. 
Proof of Lemma 5.1. We prove this lemma by induction on $|L|$.

\section{Base cases:}

Case 1: $|L|=1$. Then, $\alpha=3, \beta=\gamma=0$ and $n_{d}=1$. It can be easily seen that $|\Omega|=3<5=n_{d}-\beta-2 \gamma+5$.

Case 2: $L$ is a 3-cycle. Then, by Observation 5.4, we know that all vertices in $N(L)$ are of the same colour, say 1; and they belong to the same kempes of $\Omega$. Also, $\Omega_{2,3}$ is empty. So, $|\Omega|=2$. Furthermore, $F$ is pruned, and so $\beta=0$ and $\gamma \leq 1$. Thus, $|\Omega|=2<n_{d}-\beta-2 \gamma+5$.

\section{Induction step:}

If $L$ is a 4 -Gallai tree that does not match with any of the base cases, then (i) $L$ has a cycle $c$ that contains exactly one cut-vertex of $L$ and $c \neq L$ or (ii) $L$ has a wing $W$ and $W \neq L$.

Case 1. L has a cycle $C$ that contains exactly one cut-vertex of $L$ and $c \neq L$. By $(\mathrm{P} 1), C$ is a 3 -cycle. Let $c=\left[v_{1}, v_{2}, v_{3}\right]$, where $\operatorname{deg}_{L}\left(v_{1}\right)=3$. Also, let $a$ and $a^{\prime}$ be the vertices in $H$ that are adjacent to $v_{2}$ and $v_{3}$, respectively. By (P3), $a, a^{\prime} \in N_{\alpha}$ (and so they are distinct). By Lemma 1.5, vertices $a, a^{\prime}$ have the same colour, say 1 . Now, we consider a smaller 4-Gallai tree $L^{\prime}=L-\left\{v_{1}, v_{2}, v_{3}\right\}$, and we extend the colouring of $H$ to $v_{1}, v_{2}, v_{3}$ by assigning colours 1,2 and 3 to $v_{1}, v_{2}$ and $v_{3}$, respectively, to obtain a colouring $\phi^{\prime}$ of $F-L^{\prime}$.

It is easy to see that $F$ is a pruned $L^{\prime}$-critical graph: $v_{1} \in N_{\alpha}$ since it has exactly one neighbour in $L^{\prime}$, and so we do not violate (P2) and (P3), and furthermore, any new pendant wing must be adjacent to $v_{1}$ which is in $N_{\alpha}$, and so, $(\mathrm{P} 4)$ is not violated.

We define $\Omega^{\prime}=\left(\Omega_{1,2}^{\prime}, \Omega_{1,3}^{\prime}, \Omega_{2,3}^{\prime}\right)$ to be a collection of kempes for $\phi^{\prime}$ as 
follows: Initially, let $\Omega^{\prime}=\Omega$. Vertices $a$ and $a^{\prime}$ belong to the same kempes in $\Omega_{1,2}^{\prime}$ and $\Omega_{1,3}^{\prime}$. Now, (1) considering the path $v_{1}, v_{2}, a$ on vertices of colours 1 and 2 , we add $v_{1}$ to the kempe in $\Omega_{1,2}^{\prime}$ which contains $a$ and $a^{\prime}$, and remove $a$ and $a^{\prime}$ from it; (2) considering the path $v_{1}, v_{3}, a^{\prime}$ on vertices of colours 1 and 3 , we add $v_{1}$ to the kempe in $\Omega_{1,3}^{\prime}$ which contains $a$ and $a^{\prime}$, and remove $a$ and $a^{\prime}$ from it. Since both $a$ and $a^{\prime}$ were in the same kempes in $\Omega$, they have neighbours of both colour 2 and 3 , and thus, $a, a^{\prime} \notin P_{\phi}$. This guarantees that $\Omega^{\prime}$ is a valid collection of kempes for $\phi^{\prime}$.

In the above procedure, $n_{d}, \beta$ and $\gamma$ are not changed, and no kempe was eliminated. So, $|\Omega|=\left|\Omega^{\prime}\right| \leq n_{d}-\beta-2 \gamma+5$.

Case 2: $L$ has a wing $W \neq L$. If $L$ has no cycle, then we let $W=L-v_{0}$ where $\operatorname{deg}_{L}\left(v_{0}\right)=1$. Otherwise, by (P4), let $W \subset L$ be a wing that has a neighbour in $N_{\alpha}$ and is minimal with respect to this property.

It is easy to see that $F$ is a pruned $L^{\prime}$-critical graph: the root of $W$ is in $N_{\alpha}$ since it has exactly one neighbour in $L^{\prime}$, and so we do not violate (P2) and (P3), and furthermore, any new pendant wing must be adjacent to the root of $W$ which is in $N_{\alpha}$, and so, (P4) is not violated.

Consider an ordering of vertices in $W$, say $u_{1}, u_{2}, \ldots, u_{|W|}$, such that every vertex $u_{i}$ has exactly two neighbours in $H \cup\left\{u_{1}, \ldots, u_{i-1}\right\}$, for $1 \leq$ $i \leq|W|$. We notice that $u_{|W|}$ is the root of $W$. By the following operation MOVE-UP, we obtain a colouring $\phi^{\prime}$ of $H \cup W$ (which is an extension of $\phi$ to $W$ ) and a valid collection of kempes $\Omega^{\prime}$ for $\phi^{\prime}$.

Operation MOVE-UP: Initially, $\Omega^{\prime}=\Omega$. We colour $u_{i}$ 's in the above order as follows: Let $v_{1}$ and $v_{2}$ be the two already coloured neighbours of $u_{i}$. Vertices $v_{1}$ and $v_{2}$ have different colours as otherwise after any extension of 
the colouring to $F-u_{i}$, we can colour $u_{i}$ since it has at most two colours in its neighbourhood. So, without loss of generality, assume that $v_{1}$ has colour 1 and $v_{2}$ has colour 2. Colour $u_{i}$ with colour 3 , and remove it from $L$. Now, we (i) Add $u_{i}$ to the kempe in $\Omega_{2,3}^{\prime}$ which contains $v_{2}$. (ii) Add $u_{i}$ to the kempe in $\Omega_{1,3}^{\prime}$ which contains $v_{1}$. (iii) If any of $v_{1}$ and $v_{2}$ has no neighbour in $L$ anymore, then we delete it from the kempes.

Now, we make the following observations from Operation MOVE-UP. First we define the $\Omega$-graph of $F$ as follows: The vertex set of the kempe graph is the set of vertices in $N(L)$, and there is an edge between two such vertices if they share a kempe. Let $\Omega[W]$ denote the subgraph of the $\Omega$-graph of $F$ induced by the vertices that have at least one neighbour in $W$.

Lemma 5.6 $\Omega[W]$ is connected. Moreover, it has at least one edge to the rest of the $\Omega$-graph of $F$ or there is a vertex in $\Omega[W]$ that has a neighbour in $L-W$.

Proof. We prove this lemma by induction on $|W|$. First, consider the case when $|W|=1$, and let $v$ be the only vertex in $W$. Observation 5.5(a) implies that $\Omega[W]$ is connected. Moreover, Observation 5.5(b) implies that each neighbour of $v$ in $H$ has a neighbour in $L-W$ or it belongs to a kempe that is not contained in $\Omega[W]$, and so $\Omega[W]$ has an edge to the rest of the $\Omega$-graph.

Now, consider the case $|W|>1$. Let $u_{l}$ and $u_{r}$ be the two neighbours of the root of $W$, i.e. vertex $u_{|W|}$, in $H \cup W$. Since $|W|>1$, by symmetry we can assume that $u_{l} \in W$. Let $W_{l}$ denote the wing whose root is $u_{l}$. Now, consider an extension of the 3-colouring to $L-u_{|W|}$. Without loss of generality and by symmetry assume that $u_{l}$ and $u_{r}$ receive colours 1 and 2 , 
respectively. So, the third neighbour of $u_{|W|}$, i.e. the neighbour of $u_{|W|}$ in $L-W$, receives colour 3 . It is straightforward to show that there are unique paths on vertices of colours 1 and 2 from $u_{l}$ and $u_{r}$ to $H$. Let $v_{l}$ and $v_{r}$ be the other end of those two paths.

Notice that if $u_{r} \in H$, then $v_{r}=u_{r}$. Uncolour the vertices of $W$, and make a switch on the kempe in $\Omega_{1,2}$, namely $\omega$, that contains $v_{l}$. Again, we extend the colouring to $L-u_{|W|}$. By the choice of $v_{l}$, in this extension, vertex $u_{l}$ receives colour 2 . Consequently, in this extension, the colour of vertex $u_{r}$ must be changed to colour 1 or else $u_{|W|}$ can be coloured. So, kempe $\omega$ contains both $v_{l}$ and $v_{r}$. We consider the following two cases:

Case 1: $u_{r} \in H$. By the induction hypothesis, $\Omega\left[W_{l}\right]$ is connected. Now, $\Omega[W]$ is connected since $V(\Omega[W])=V\left(\Omega\left[W_{l}\right]\right) \cup\left\{u_{r}\right\}$ and kempe $\omega$ contains both $u_{r}$ and $v_{l}$ which is adjacent to $W_{l}$.

Case 2: $u_{r} \in W$. Let $W_{r}$ denote the wing whose root is $u_{r}$. By the induction hypothesis, $\Omega\left[W_{l}\right]$ and $\Omega\left[W_{r}\right]$ are connected. Now, $\Omega[W]$ is connected since $V(\Omega[W])=V\left(\Omega\left[W_{l}\right]\right) \cup V\left(\Omega\left[W_{r}\right]\right)$ and $\omega$ contains both $v_{l}$ and $v_{r}$ which are adjacent to $W_{l}$ and $W_{r}$, respectively.

Finally, by considering the unique path on vertices of colours 1 and 2 from $u_{l}$ to $H$, the same argument proves that there is a kempe that contains a vertex that is adjacent to $W$ and a vertex that is adjacent to $L-W$ (which implies that $\Omega\left[W_{l}\right]$ has at least one edge to the rest of the $\Omega$-graph), or there is a vertex in $H$ that has neighbours in both $W$ and $L-W$.

Let $\beta_{i}$ and $\gamma_{i}$ be the number of vertices in $N_{\beta}$ and in $N_{\gamma}$, respectively, with exactly $i$ neighbours in $W$ before Operation MOVE-UP. Let $\alpha_{1}$ denote the number of vertices in $N_{\alpha}$ with (exactly) one neighbour in $W$ before 
Operation MOVE-UP. The tree structure of $W$ implies that

$$
\alpha_{1}+\sum_{i \geq 1} i\left(\beta_{i}+\gamma_{i}\right)=|W|+1
$$

We observe that vertices in $N_{\beta}$ with $<2$ neighbours in $W$ and those in $N_{\gamma}$ with $<4$ neighbours in $W$ have neighbours in $L-W$ (by (P2) and Lemma 4.1), and so, will not be deleted from the collection of kempes. Let $k$ be the number of kempes deleted during operation MOVE-UP. Notice that a vertex in $N_{\gamma}$ with exactly one neighbour in $W$ will be moved to $N_{\beta}$ after operation MOVE-UP. Now, by the induction hypothesis, we have

$$
\begin{aligned}
|\Omega| & =\left|\Omega^{\prime}\right|+k \\
& \leq\left(n_{d}-|W|\right)-\left(\beta+\gamma_{1}-\sum_{i \geq 1} \beta_{i}\right)-2\left(\gamma-\sum_{i \geq 1} \gamma_{i}\right)+5+k \\
& \leq n_{d}-\beta-2 \gamma+\sum_{i \geq 1} \beta_{i}+\gamma_{1}+2 \sum_{i \geq 2} \gamma_{i}-|W|+5+k \\
& \leq n_{d}-\beta-2 \gamma+5+k-\left(\alpha_{1}-1+\sum_{i \geq 2}(i-1) \beta_{i}+\sum_{i \geq 3}(i-2) \gamma_{i}\right)
\end{aligned}
$$

where the last inequality is obtained by applying (9). Then it suffices to show that

$$
k \leq \alpha_{1}-1+\sum_{i \geq 2}(i-1) \beta_{i}+\sum_{i \geq 3}(i-2) \gamma_{i} .
$$

In order to prove (11), we use what we call the deletion graph of $W$. The vertex set of the deletion graph is the set of vertices that belong to at least one deleted kempe. There is an edge between two such vertices if they share a deleted kempe. Two vertices have two edges between them when they share both their (deleted) kempes.

The right hand side of (11) is a trivial upper bound on the number of vertices of the deletion graph minus 1. To get a feeling for the deletion graph, 
we first show that it is fairly easy to prove a relaxed version of (11). Let $M$ be a component of the deletion graph. Let $\beta_{i}(M)$ and $\gamma_{i}(M)$ denote the number in $M$ of vertices in $N_{\beta}$ and in $N_{\gamma}$, respectively, with exactly $i$ neighbours in $W$ before Operation MOVE-UP. Let $\alpha_{1}(M)$ denote the number in $M$ of vertices in $N_{\alpha}$ with (exactly) one neighbour in $W$ before Operation MOVEUP. Similarly, let $k(M)$ be the number of kempes that are deleted during Operation MOVE-UP and consist only of vertices in $M$.

$$
k(M) \leq|M|+1 \leq \alpha_{1}(M)+\sum_{i \geq 2} \beta_{i}(M)+\sum_{i \geq 4} \gamma_{i}(M)+1 .
$$

To see the right hand side inequality, notice that to count the number of deleted kempes after $W$ to $H$, we only need to consider those kempes that contain only vertices which have no neighbours in $L-W$. So, those kempes that contain vertices that are counted in $\beta_{1}, \gamma_{1}, \gamma_{2}$ or $\gamma_{3}$ (by (P2), and Lemma 4.1) will not be deleted. To see $k(M) \leq|M|+1$, we consider a rooted spanning tree of $M$. The root of the tree may be involved in at most two deleted kempes. Now, each child of the root is also involved in at most two deleted kempes. However, since it has an edge to its parent, one of the child's kempes is in common with its parent and has already been considered. So, the child adds only one to the number of deleted kempes. Similarly, every vertex other than the root adds at most one to the number of deleted kempes, and this implies (12).

Now, consider a less relaxed version of (12):

$$
k(M) \leq \alpha_{1}(M)+\sum_{i \geq 2}(i-1) \beta_{i}(M)+\sum_{i \geq 3}(i-2) \gamma_{i}(M) .
$$

(12) is tight only if every kempe that intersects with the vertices of $M$ is deleted. Since $M$ is a component of the deletion graph of $W$, no vertex 
of $M$ has a neighbour in $L-W$. Now, since $W \subset L$, by Lemma 5.6, there is an edge in the kempe graph that intersects $M$ but is not in $M$. Thus, $M$ is a proper subgraph of the $\Omega$-graph, and so, (13) is proved.

Remark 5.7 In fact, we just proved that $k(M) \leq|M|$. Since $k(M) \leq$ $\alpha_{1}(M)+\sum_{i \geq 2} \beta_{i}(M)+\sum_{i \geq 3} \gamma_{i}(M)$, inequality (13) is tight only if $\beta_{i}(M)=0$ and $\gamma_{i}(M)=0$ for all $i>2$.

Now, by summing up (13) over all the components of the deletion graph of $W$, we obtain

$$
k \leq \alpha_{1}+\sum_{i \geq 2}(i-1) \beta_{i}+\sum_{i \geq 3}(i-2) \gamma_{i}
$$

Remark 5.8 To complete the proof of (11) it suffices to show that (13) is not tight for at least one of the components of the deletion graph.

Now, we complete the proof of Lemma 5.1 by considering each possible choice of $W$.

Case $i: W=L-v_{0}$ where $\operatorname{deg}_{L}\left(v_{0}\right)=1$. By (14), we get $k \leq \alpha_{1}+$ $\sum_{i \geq 2}(i-1) \beta_{i}+\sum_{i \geq 3}(i-2) \gamma_{i}$. So, we have $|\Omega|=\left|\Omega^{\prime}\right|+k \leq 3+k \leq$ $3+\alpha_{1}+\sum_{i \geq 2}(i-1) \beta_{i}+\sum_{i \geq 3}(i-2) \gamma_{i}$ where the first inequality is from Case 1 of the base cases. Now, let $\beta(W)=\sum_{i \geq 2} \beta_{i}$ and $\gamma(W)=\sum_{i \geq 3} \gamma_{i}$. The two neighbours of $v_{0}$ in $H$ are the only two vertices that may have more neighbours in $L$ than what they have in $W$. So, we have $\beta+2 \gamma \leq \beta(W)+$ $2 \gamma(W)+2$. Thus, by (9) we get $|\Omega| \leq 3+|W|+1-\beta-2 \gamma+2=n_{d}-\beta-2 \gamma+5$ since $n_{d}=|W|+1$.

Recall that by the choice of $W$, if $W$ does not satisfy Case i, then $W$ has a neighbour in $N_{\alpha}$ and is minimal with respect to this property. So, we 
have one of the following two cases:

Case ii: $W$ is adjacent to two vertices in $N_{\alpha}$. By the minimality of $W$, we have $|W|=1$. More precisely, $W$ is a single vertex which is adjacent to two vertices in $N_{\alpha}$. Thus, Operation MOVE-UP does not change $\beta$ and $\gamma$, and will delete at most one kempe. So, $|\Omega| \leq\left|\Omega^{\prime}\right|+1 \leq\left(n_{d}-1\right)-\beta-2 \gamma+5+1 \leq$ $n_{d}-\beta-2 \gamma+5$ by the induction hypothesis.

Case iii: $W$ is adjacent to exactly one vertex in $N_{\alpha}$. Recall from Remark 5.8 that in order to prove (11), it suffices to prove that for at least one of the components of the deletion graph of $W,(13)$ is not tight. Let $M$ be the component of the deletion graph that contains the vertex in $N_{\alpha}$, say $v$. Recall that by Remark 5.7, inequality (13) is tight for $M$ only if $M$ does not contain any vertex other than $v$ or those counted in $\beta_{2}(M)$ (i.e. if $\alpha=1$, $\beta_{i}(M)=0$ and $\gamma_{i}(M)=0$ for all $i>2$ ). Now, let $\omega$ be a deleted kempe that contains $v$. (If there is no such kempe then we are already done since $v$ forms a component $M$ where $k(M)=0$, and so (13) is not tight.) We claim that the number of edges from the vertices in $\omega$ to the wing $W$ was even before the operation. We show this by proving that Operation MOVE-UP does not change its parity: in Operation MOVE-UP, steps (i) and (ii) do not change the number of edges from the vertices in $\omega$ to the wing $W$. Moreover, if $v$ is deleted from the kempes, then Observation 5.5 implies that it does not form a kempe on its own. Therefore, step (iii) eliminates $\omega$ only if $\omega=\left\{v, v^{\prime}\right\}$ where $v, v^{\prime} \in N_{\alpha}$ at the beginning of the iteration, and so, step (iii) deducts exactly 2 from $\omega$. This is not possible if $v \in \omega$ and the rest of the vertices of $\omega$ have exactly two neighbours in $W$. Thus, at least one vertex of $\omega$ other than $v$ has odd number of neighbours in $W$, and so $\beta_{i}(M)>0$ or $\gamma_{i}(M)>0$ 
for some $i>2$. So, (13) cannot be tight for $M$. This implies (11).

\section{$6 \quad$ Extending our main theorem}

We believe that, with sufficient labour, the proof of Theorem 1.2 can be extended to $k$-critical graphs with connected low-vertex subgraphs for $k=5$ and perhaps for greater values of $k$. However, a more important goal would be to extend Theorem 1.2 to 4 -critical graphs with disconnected low-vertex subgraphs, and thus prove Gallai's Conjecture for the case $k=4$. The main difficulty is that when $L$ has more than one component, we don't have nearly as much control on the colours that can appear on the vertices of $N(L)$ (i.e. those adjacent to $L)$ in 3 -colourings of $H$.

For example, in the simplified setting of Special Case 2.1, i.e. where every vertex of $L$ lies in a cycle of $L$, it was easy to show that in any 3 -colouring of $H$, the vertices adjacent to $L$ all have the same colour. However, for disconnected $L$, it is only true that the vertices adjacent to one component of $L$ must all have the same colour.

Our restrictions on the permissable colourings of $N(L)$ allowed us (essentially) to say something about the extent to which the vertices of $N(L)$ must be joined by kempe chains, and those kempe chains contributed many edges to $H$. The lack of similar restrictions has thus far prevented us from extending these arguments to the case where $L$ is disconnected.

On the other hand, we close with an observation that may make things a bit simpler for the case where $L$ is disconnected: In that case, we can assume that we are in the simplified setting of Special Case 2.1. In fact, 
using the Hajos Construction, one can show that we only need to consider Gallai forests where each component consists of two triangles joined by an edge.

\section{Acknowledgement}

We are grateful to Michael Steibitz for several helpful comments on this proof. We are also grateful to two anonymous referees for many useful comments that substantially improved the presentation of this paper.

\section{References}

[Dir57] G. A. Dirac. A theorem of R.L. Brooks and a conjecture of H. Hadwiger. Proc. London Math. Soc., 3(7):161-195, 1957.

[Dir74] G. A. Dirac. The number of edges in critical graphs. J. Reine Angew. Math., 268/269:150-164, 1974. Collection of articles dedicated to Helmut Hasse on his seventy-fifth birthday, II.

[Far05] B. Farzad. On colourings of graphs. Thesis (PhD), University of Toronto, 2005.

[Gal63] T. Gallai. Kritische Graphen. I. Magyar Tud. Akad. Mat. Kutató Int. Közl., 8:165-192, 1963.

[JT95] T. R. Jensen and B. Toft. Graph coloring problems. John Wiley \& Sons Inc., New York, 1995. A Wiley-Interscience Publication.

[KM72] H. V. Kronk and J. Mitchem. On Dirac's generalization of Brooks' theorem. Canad. J. Math., 24:805-807, 1972. 
[Kri98] M. Krivelevich. An improved bound on the minimal number of edges in color-critical graphs. Electron. J. Combin., 5(1):Research Paper 4, 4 pp. (electronic), 1998.

[KS98] A. V. Kostochka and M. Stiebitz. Colour-critical graphs with few edges. Discrete Math., 191(1-3):125-137, 1998. Graph theory (Elgersburg, 1996).

[KS99] A. V. Kostochka and M. Stiebitz. Excess in colour-critical graphs. In Graph theory and combinatorial biology (Balatonlelle, 1996), pages 87-99. János Bolyai Math. Soc., Budapest, 1999.

[KS03] A. V. Kostochka and M. Stiebitz. A new lower bound on the number of edges in color-critical graphs and hypergraphs. J. Combin. Theory Ser. B, 87(2):374-402, 2003.

[Mit78] J. Mitchem. A new proof of a theorem of Dirac on the number of edges in critical graphs. J. Reine Angew. Math., 299/300:84-91, 1978.

[Sti82] Michael Stiebitz. Proof of a conjecture of T. Gallai concerning connectivity properties of colour-critical graphs. Combinatorica, $2(3): 315-323,1982$.

[Wei75] J. Weinstein. Excess in critical graphs. J. Combinatorial Theory Ser. B, 18:24-31, 1975. 